\title{
Genomic evolution of the human and animal coronavirus diseases
}

\author{
Carla Miranda ${ }^{1,2} \cdot$ Vanessa Silva ${ }^{1,2,3,4} \cdot$ Gilberto Igrejas $^{2,3,4} \cdot$ Patrícia Poeta $^{1,2}$ (1)
}

Received: 6 May 2021 / Accepted: 5 August 2021 / Published online: 12 August 2021

(c) The Author(s), under exclusive licence to Springer Nature B.V. 2021

\begin{abstract}
Different coronaviruses have emerged due to their ability to infect, mutate and recombine multiple species and cell types, suggesting that these viruses will carry on to evolve and origin both veterinary and human diseases. So far, more than fifteen coronavirus-related diseases have been described in animals and seven in humans. Of which recently, a novel human betacoronavirus designated by severe acute respiratory syndrome coronavirus 2 (SARS-CoV-2), an emerging zoonotic coronavirus is the causative agent of the coronavirus disease 2019 . This virus emerged in China and spread rapidly worldwide. At the end of January 2020, the WHO declared the pandemic as a public health emergency of international concern. In this pandemic, the SARS-CoV-2 virus has infected more than 198 million people, with 4.2 million deaths worldwide (as of 2 August 2021). In the past two decades, this is the third betacoronavirus that has crossed the interspecies barrier from animals to infect humans and other animal species. The diseases caused mainly severe respiratory infections. The aim of this review is to summarize and provide an overview of the coronaviruses that can affect animals and humans and the diseases that ensue, as well as, its genomic relationship.
\end{abstract}

Keywords Coronavirus $\cdot$ Animal coronaviruses $\cdot$ Human coronaviruses $\cdot$ SARS-CoV-2 $\cdot$ COVID-19

\section{Introduction}

Coronaviruses (CoVs) belong to the subfamily Orthocoronavirinae, part of the family Coronaviridae within the order Nidovirales. This subfamily is divided into the four genera Alpha, Beta, Delta and Gammacoronavirus, according to the International Committee on Taxonomy of Viruses (ICTV). The CoVs are enveloped positive-sense RNA viruses, with large surface projections shaped like clubs or petals called spikes. Electron microscope images of the virus are reminiscent of the sun's corona, hence the name coronavirus

Patrícia Poeta

ppoeta@utad.pt

1 Microbiology and Antibiotic Resistance Team (MicroART), Department of Veterinary Sciences, University of Trás-OsMontes and Alto Douro, Vila Real, Portugal

2 Associated Laboratory for Green Chemistry (LAQV-REQUIMTE), University NOVA of Lisboa, Lisboa, Caparica, Portugal

3 Department of Genetics and Biotechnology, University of Trás-Os-Montes and Alto Douro, Vila Real, Portugal

4 Functional Genomics and Proteomics Unit, University of Trás-Os-Montes and Alto Douro, Vila Real, Portugal
[1]. All coronavirus genomes are arranged similarly and are the largest known RNA genomes $(27-32 \mathrm{~kb})$. The replicase locus is encoded within the $5^{\prime}$ region of the genome that included two overlapping open reading frames [ORFs 1a and $1 \mathrm{~b}$ with a ribosomal frameshift (RFS) site and several nonstructural proteins (nsp)] and the structural proteins within the 3 ' region. Structural proteins comprise spike (S), envelope $(\mathrm{E})$, membrane $(\mathrm{M})$, nucleocapsid $(\mathrm{N})$ and internal proteins, as well as hemagglutinin esterase glycoprotein in the betacoronaviruses (forming a second type of surface projection). Structural proteins with functions in virion structure and morphogenesis contribute significantly to viral spread in vivo and in antagonizing host cell responses. Conserved nonstructural proteins also have functions in antagonizing host responses and enzymatic activities in RNA metabolism or protein processing $[1,2]$.

The RNA viruses, in particular with non-segmented genomes and positive-strand, have demonstrated the ability to evolve quickly facing the changing host environments and/or ecological conditions. The RNA viruses balance the capacity to adapt to new situations and the need to maintain an intact replication-competent genome. Generally, there is an inverse correlation between genome size and replication fidelity as a larger genome is more impacted by high error 
rates during RNA replication and high recombination frequencies. Therefore, these viruses present nucleotide substitution rates higher than those observed in DNA viruses $[2,3]$.

In the last 50 years, many different coronaviruses causing a wide variety of human and veterinary diseases have emerged due to their ability to recombine, mutate and infect multiple species and cell types. It is indeed likely that these viruses will continue to emerge, evolve and origin both veterinary and human disease outbreaks. Coronaviruses infect certain domestic and wild animals and humans, causing acute or chronic diseases (Fig. 1), some of which may be subclinical or asymptomatic $[2,4,5]$.

\section{Animal coronaviruses}

The CoVs include a substantial number of pathogens of mammals and birds that cause severe disease mainly in livestock (pigs, cows and chickens) and companion animals (dogs and cats) (Fig. 1). Ducks, turkeys, horses, bats, snakes, mice, ferrets, minks and other wild animals are also known to have been infected by different CoVs. Alpha and Betacoronavirus species are mostly responsible for the numerous diseases that ensue including respiratory disease, enteritis, polysevositis, myocarditis, sialodacryadenitis, hepatitis, encephalomyelitis, nephritis, immunopathological diseases and various other disorders $[2,4,5]$.

In brief, more than fifteen CoV-related diseases have been identified in animals, but with different tissue tropisms and consequently different clinical signs. Some examples will be described here. Porcine epidemic diarrhea virus (PEDV) and transmissible gastroenteritis virus (TGEV) both included in Alphacoronavirus genus, cause severe gastroenteritis in young piglets, leading to significant morbidity, mortality and economic losses. Similar to these swine enteric pathogens, in the last decade emerged the porcine deltacoronavirus (PDCoV) of the Deltacoronavirus genus, affecting also neonatal piglets with diarrhea, vomiting and dehydration. Porcine hemagglutinating encephalomyelitis virus (PHEV) belonging to Betacoronavirus genus mostly leads to enteric infection but can also infect the nervous system of pigs, causing encephalitis, vomiting and wasting [4-6]. Feline enteric coronavirus $(\mathrm{FCoV})$ is highly contagious with
Fig. 1 List of main virus species belong to the Coronaviridae family that affect livestock, pets or humans, according to the International Committee on Taxonomy of Viruses

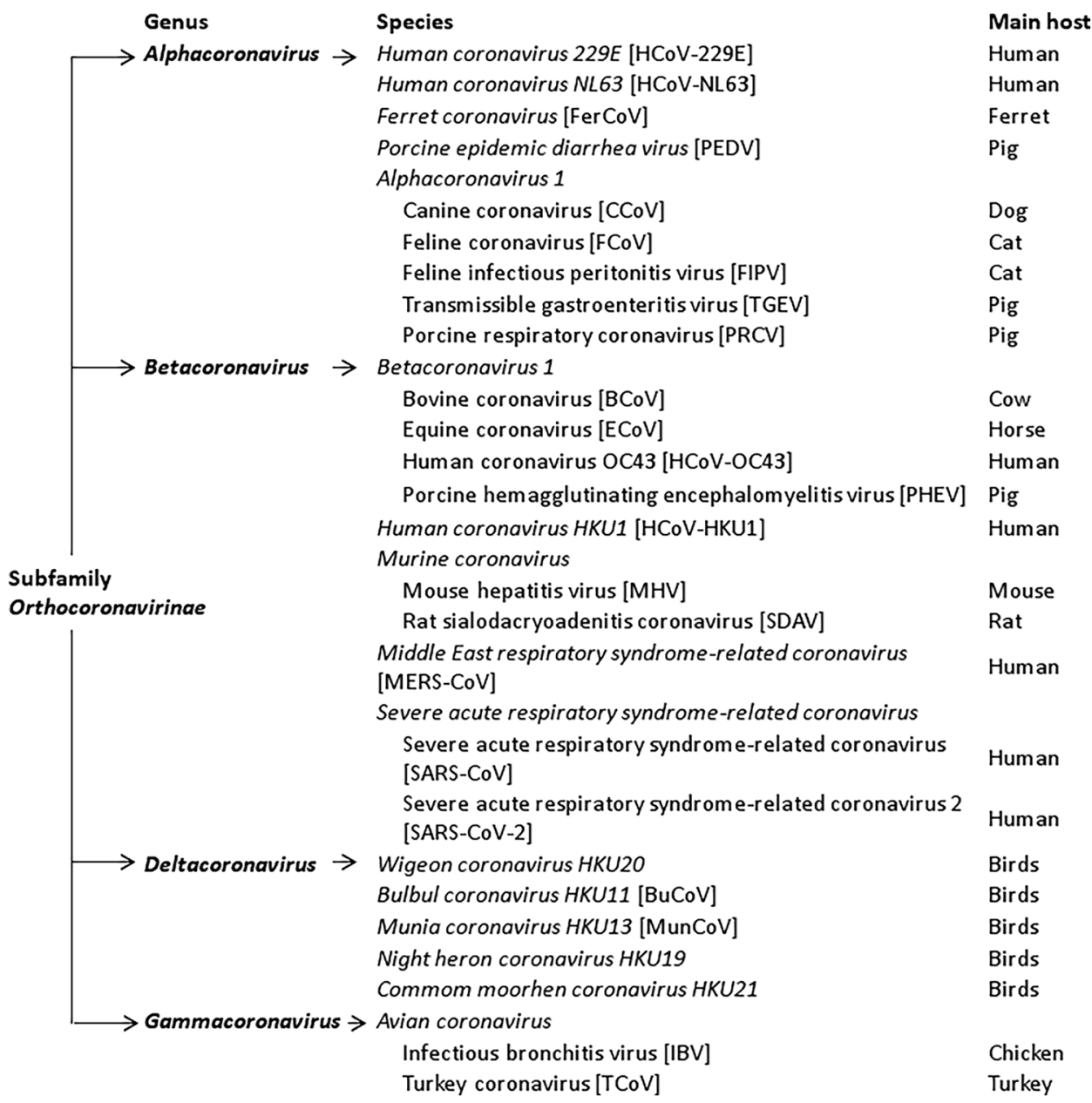


fecal-oral transmission and this can cause mild or asymptomatic infections, in opposite feline infectious peritonitis (FIP), a lethal disease in domestic cats. FIP occurs in wet and dry forms with similarities to the human disease sarcoidosis. A canine coronavirus $(\mathrm{CCoV})$ usually only produces mild gastroenteritis in infected dogs but it has been identified in foxes, raccoon dogs and cats [4, 5, 7].

Bovine coronavirus $(\mathrm{BCoV})$ infections are associated with three distinct clinical syndromes in cattle: diarrhea in calves, winter dysentery or hemorrhagic diarrhea in adult cattle, and respiratory infections in cattle of various ages, including the bovine respiratory disease complex (or shipping fever) in feedlot cattle. The $\mathrm{BCoV}$ infects cattle and wild ruminants, and are associated with a significant economic loss to the livestock industry. Many strains of the rat coronavirus sialodacryoadenitis virus (SDAV) are highly contagious within naïve rat populations with primary tropism to nasal respiratory epithelium, then to lacrimal glands, salivary glands and lungs $[4,5]$. Infectious bronchitis virus (IBV) causes one of the major viral diseases of chickens worldwide. This virus spreads between birds by aerosol and ingestion of food contaminated with feces, causing gasping, coughing, tracheal rales, sneezing, nasal exudate, wet eyes, respiratory distress, and occasionally swollen sinuses. As a result of the extensive use of vaccines this disease has declined [5].

The most widely studied animal coronavirus is murine coronavirus mouse hepatitis virus (MHV), which causes a variety of outcomes in mice, including respiratory, enteric, hepatic and neurological infections. These infections often serve as useful models of human and animal disease. MHV represents a spectrum of coronaviruses with tropisms for a wide variety of mouse cells or tissues, such as the enteric epithelium, respiratory epithelium, vascular endothelium, lymphoid tissue, hemopoietic tissue, liver and central nervous system $[4,5,8]$.

\section{Human coronaviruses}

Coronaviruses are among the spectrum of viruses that cause the common cold or mild respiratory tract infections in humans. Four human coronaviruses ( $\mathrm{HCoVs}$ ), namely HKU1, NL63, 229E and OC43, have been circulating in the population, generally causing mild respiratory disease (Fig. 1). In severe cases, these viruses can also cause pneumonia and bronchiolitis in children, the elderly, or immunecompromised individuals $[9,10]$. The HCoV-229E (genus Alphacoronavirus) and HCoV-OC43 (genus Betacoronavirus) were identified in 1967 and are responsible for $10-30 \%$ of all common colds and infections occurring mainly during the winter and early spring [11]. In winter 2002-2003 in the Netherlands, HCoV-NL63 (genus Alphacoronavirus) was isolated from a child with bronchiolitis and conjunctivitis [12]. In winter 2003-2004 in China, HCoV-HKU1 (genus Betacoronavirus) was discovered in two patients with pneumonia [12]. The natural hosts of all HCoVs are animals, such as bats for Alphacoronavirus and rodents for Betacoronavirus, and intermediate hosts are known to be camelids for $\mathrm{HCoV}-229 \mathrm{E}$ and bovine for $\mathrm{HCoV}-\mathrm{OC} 43[9,13]$.

In 2002, before HCoV-NL63 and HCoV-HKU1 were identified, a new betacoronavirus called severe acute respiratory syndrome-related coronavirus (SARS-CoV) emerged in the human population in China as an atypical pneumonia, causing a worldwide epidemic with severe morbidity and high mortality rates, particularly in older individuals. The concept that the human coronaviruses only cause common colds completely changed with the emergence of this highly pathogenic zoonotic disease, severe acute respiratory syndrome (SARS). The causal agent, SARS-CoV, had its origin in bats but crossed over to humans via an intermediate host, the masked palm civet sold in a market of live animals, the possible source of human infections. Closely related viruses were since isolated from several exotic animals including Himalayan palm civets and raccoon dogs [4, 14, 15]. Although cats and ferrets can be infected with SARS-CoV, the infection does not induce severe disease in either species [16]. During the 2002-2003 outbreak, SARS-CoV affected approximately 8500 people and led to 900 deaths, resulting in an overall mortality rate of $9-11 \%$ but approaching $50 \%$ mortality in individuals over 60 years of age. The outbreak began in a hotel in Hong Kong and spread to 29 countries/ regions $[14,17]$. However, the virus has not returned since this outbreak [4].

In 2012, a new betacoronavirus was transmitted from animals to humans causing the lethal Middle East respiratory syndrome (MERS). The MERS-related coronavirus (MERS$\mathrm{CoV}$ ) emerged in Saudi Arabia through contact with dromedary camels as the intermediate host although it originated from bats. MERS-CoV went on to affect around 2500 people and caused 858 deaths, with a mortality rate of $35 \%$. This respiratory disease is particularly severe and, unlike SARS$\mathrm{CoV}$, sporadic MERS outbreaks have been reported in the interim $[18,19]$.

At the end of December 2019, a new outbreak of a respiratory disease infecting humans in Wuhan City in the Hubei province of China was reported. Clinically, patients had symptoms and signs similar to SARS and MERS, such as fever, coughing and difficulty breathing, with evolution to severe pneumonia in the most severe cases, which needed ventilator support $[13,15,20]$. A few days after the outbreak was announced, scientists identified a novel betacoronavirus by molecular analysis, provisionally called 2019-nCoV [20, 21]. According to the Coronaviridae Study Group (CSG) of the ICTV, based on phylogeny, taxonomy and established practice, the virus was designated SARS-CoV-2, since this 
created a sister clade to the prototype bat and human SARSCoVs. The ongoing outbreak caused by coronavirus-associated acute respiratory disease was called coronavirus disease 19 (COVID-19) [22].

From the evolution of an animal betacoronavirus to humans, COVID-19 is the third documented spillover that has resulted in a pandemic in the last 20 years [23]. SARSCoV-2 also originated in bats and was transmitted to humans through yet unknown animals in Wuhan, although pangolins and snakes are possible intermediary hosts [10, 20, 21]. Since a bat coronavirus (Bat-CoV-RaTG13) that has been isolated from Yunnan, China shares 96\% whole genome identity with SARS-CoV-2 and another coronavirus was recently isolated from Malayan pangolins, demonstrating that the whole genomic sequence of the pangolin coronavirus (pangolin-CoV) is $91.02 \%$ and $90.55 \%$ identical to that of SARS-CoV-2 and bat-CoV-RaTG13, respectively [21, 24]. Human outbreaks are driven by person-to-person contact. The animal susceptibility with humans in close contact was searched, demonstrating that SARS-CoV-2 replicates weakly in dogs, ducks, chickens and pigs. However, cats and ferrets are permissive to infection and cats are susceptible to airborne infection [23]. Currently, there is no evidence that infected pets are a source of infection for people or other pets, suggesting infection was only caused by human-toanimal transmission [25]. Several dogs and felines (domestic cats and a tiger) have tested positive for SARS-CoV-2 after close contact with infected humans, as reported by the World Organization for Animal Health (OIE) [26]. However, pets are unlikely to be SARS-CoV-2 carriers, since companion animals including pet dogs (including one dog a SARS-CoV-2 patient kept and two dogs which had close contact with it) and cats, street dogs and cats were serologically negative for the SARS-CoV-2 virus [27].

COVID-19 is a respiratory disease that affects people of all ages, most of whom will develop mild to moderate symptoms and recover without requiring special treatment. People who have underlying medical conditions and those over 60 years old have a higher risk of developing severe disease and death [28]. Many people are asymptomatic. The disease is transmitted by inhalation or contact with infected droplets and the incubation period ranges from 2 to 14 days [10]. The disease is rapidly progressing worldwide and the mortality rates are high. At the end of January 2020, the World Health Organization (WHO) declared a Public Health Emergency of International Concern. Since the identification of the outbreak the infection quickly spread, resulting in more than 198 million confirmed cases and 4.2 million deaths from 216 countries, areas or territories with cases as of 02 August 2021 [28]. These numbers possibly underestimate the total number of infected and dead, as many people are likely to be asymptomatic carriers, while testing is limited and surveillance is variable. Owing to the lack of effective therapeutics to date, the best measures to control human coronaviruses remain a strong public health surveillance system coupled with rapid diagnostic testing and quarantine when necessary [4]. However, a year after the pandemic began, several vaccines were developed and marketed, leading to a mass and rapid human vaccination program starting in December 2020 [28]. Diagnosis of SARS-CoV-2 is based mainly on respiratory secretions on detection of the virus by molecular tests like nucleic acid amplification testing [10] and viral proteins by antigenic tests called direct immunoassay detection of viral protein antigens. Although this last allows a rapid antigen-detecting, it demonstrates a lower sensitivity than molecular tests [29]. The infection spreads so fast and the level of morbidity is so high that to control outbreaks public health organizations need to rapidly identify cases and strictly implement appropriate control measures [30]. The measures to prevent transmission entail home isolation of suspected cases and those with mild illnesses, standard precautions including hand hygiene and strict infection control measures at hospitals that include contact and droplet precautions with the use of personal protective equipment $[10,31]$.

However, most people with COVID-19 (84\%) develop a mild or uncomplicated illness no requiring hospital interventions. Some develop severe disease requiring hospitalization and oxygen support (14\%) with 5\% requiring admission to an intensive care unit (ICU), in which most of critically ill patients require mechanical ventilation [32]. In severe cases, it might be necessary to proactively prevent complications, secondary infections, treat underlying diseases and provide organ function support, such as acute respiratory disease syndrome (ARDS), severe acute respiratory infection (SARI), sepsis and septic shock and multi-organ failure, including acute kidney injury, liver dysfunction and cardiac injury $[33,34]$. The median duration of viral RNA detection was 20 days in survivors and the longest duration of viral shedding in survivors was 37 days. Additionally, SARS$\mathrm{CoV}-2$ virus was detectable until death in non-survivors $[31,35,36]$.

According to the WHO guidelines for clinical management of patients with COVID-19, the treatment is essentially supportive and symptomatic [31]. Supportive treatment includes bedrest, adequate nutrition, monitoring vital signs and oxygen saturation, prevention of dehydration and maintaining water, electrolyte and acid-base balance [33]. There is no current evidence to recommend any specific anti-COVID-19 treatment for patients with confirmed COVID-19. While a variety of antimicrobials agents, including antiviral peptides and corticosteroids, antibiotics (macrolides and tetracycline) and antifungals have been used in COVID-19 patients, their role and mode of action have yet to be established. For example, based on some positive experiences with SARS and MERS, antiviral drugs such as 
ribavirin and lopinavir-ritonavir have been used [11, 37, 38]. There are many ongoing clinical trials testing various potential antivirals (registered on https://clinicaltrials.gov/) [39].

\section{Genomic relationship among the different coronaviruses}

The scientific community has searched in diverse areas to understand the viral-host interactions to work in treatment alternatives and stop the disease progression mainly since the COVID-19 pandemic began, although there are nongenetic and genetic factors associated with these interactions. From the epigenetic studies is possible to evaluate the mechanisms as the virus interferes with the host immune response and quickly spread infection [40]. Saksena et al. [40] highlighted the importance of host-virus interactions through the SARS-CoV-2 epigenetics, however, coronavirus pathogenesis results from the complex epigenetic mechanisms and depends on the virus ability to enter, replicate and affect the host immune system. These mechanisms that modulate and regulate host gene expression include histone modifications chromatin remodeling, non-coding RNAs, and DNA methylation.

For many years, the CoVs have been known in both animal and human medicine. CoVs posse large genomes and show an exceptional genetic plasticity with rapid genetic evolution and a change of the antigenic profile, demonstrating adaption to a variety of new hosts or reservoirs and tissue tropism. These characteristics result of the viral replicase mechanism, such as RNA dependent-RNA polymerase ( $p o l)$ and helicase (Hel), due to the inclusion of error nucleotides at each replication cycle, accumulating changes or mutations in the viral genome or the recombination mechanism allowing the CoVs acquire new biological properties [41]. In coronaviruses, the selection of $\mathrm{CpG}$ suppressed clones and cytosine deamination are the main biological factors responsible by codon usage bias. Recombination was frequently observed between different strains of the same $\mathrm{CoV}$ or other CoVs [42].

In general, the described ideal host for Alpha- and Betacoronaviruses are mammals, bats, and rodents, including several zoonotic and human CoVs, and the Gamma- and Deltacoronaviruses are mainly detected in birds, demonstrating some genomic differences among these genera. Although all CoVs present a similar organization of the genome, some conserved genes present additional ORFs [42-44]. For example, the CSG analyzes only five essential domains like $3 \mathrm{CL}^{\text {pro }}, \mathrm{NiRAN}, \mathrm{RdRp}, \mathrm{ZBD}$ and HEL, the only ones conserved in all viruses of the order Nidovirales, to classify the $\mathrm{CoVs}$ including the SAR-CoV-2 [22]. Based on phylogenetic analysis and bioinformatic methods, is allowed to distinguish the Alphacoronaviruses of other CoV genera (Figs. 2 and 3).
This group shows a transcription regulatory sequence (TRS) that occurs by the polymerase jumping with the sequence 5'-CUAAAC-3'. The TRS can be found in the genome at the $5^{\prime}$ end of each gene and $3^{\prime}$ end of the leader sequence. In addition, all strains contain one or two nonstructural proteins (NS7a and b) downstream to $\mathrm{N}$ and two papain-like proteases $\left(\mathrm{PL}^{\text {pro }}\right.$ and PL2 $\left.{ }^{\text {pro }}\right)$ in nsp3 of ORF1ab $[42,45]$. A major of AlphaCoV members possess one ORF3 between the $\mathrm{S}$ and $\mathrm{E}$ genes, while FCoVs and CCoVs contain three (ORF3a, 3b and 3c) and TGEV two (ORF3a and 3b) with unknown functions [7].

All members of Betacoronaviruses possess two papainlike proteases ( $\mathrm{PL}^{\text {pro }}$ and PL2 ${ }^{\text {pro }}$ ), haemagglutinin esterase (HE) genes and the $S$ proteins are cleaved into the $S 1$ and S2 domains, except for SARS-CoV and bat-CoVs that only contain one $\mathrm{PL}^{\text {pro }}$, no encode $\mathrm{HE}$, unique $\mathrm{S}$ domain and the sequence for the TRS is ACGAAC in the E gene instead of CUAAAC. From the CoV genomes, the proteins that demonstrate more variability is the $\mathrm{S}$ proteins, and when there are two domains from these, the $\mathrm{S} 1$ domain is the most variable. Additionally, the sequenced SARS-CoV genome isolated from humans and civet showed $>95 \%$ amino acid similarity of the pol (nsp12), helicase (nsp13) and $\mathrm{N}$ genes relative to bat SARS-CoV. However, the S protein only shared approximately $80 \%$ of similarity, suggesting that this variability in SARS-CoV protein $\mathrm{S}$ has allowed interspecies transmission $[42,46]$. The SARS-CoV-2 genome shows similarity to SARS-CoVs, containing the SARS-CoV-2 eight accessory proteins in the 3 '-terminus, 3a, 3b, p6, 7a, 7b, $8 \mathrm{~b}, 9 \mathrm{~b}$ and orf 14; however, in SARS-CoV the 8 a protein is present and the $3 \mathrm{~b}$ is longer. Additionally, the observed amino acid substitutions occurred in nsp2 and nsp3, and S gene [47].

The Gammacoronaviruses genus includes IBV and recently turkey coronavirus ( $\mathrm{TCoV}$ ) that has been identified in several birds species; both sequenced genomes were similar relative to $\mathrm{G}+\mathrm{C}$ contents, organization and size, suggesting that both have diverged from the same ancestor. In all members also present two domains of the $S$ proteins [46]. Gamma- and Deltacoronaviruses lack nsp1 in the ORF1a polyprotein, in which the gene 1 only encodes 15 nsps. However, the nsp1 in the other two genera show different functions and characteristics, suggesting an important role in the adaptation of CoVs to different host species and in evolutionary relatedness [42, 48]. In 2012, seven novel Deltacoronaviruses were identified in pigs and birds, comprises porcine coronavirus HKU15, wigeon coronavirus HKU20, magpie robin coronavirus HKU18, whiteeye coronavirus HKU16, sparrow coronavirus HKU17, night heron coronavirus HKU19, and common moorhen coronavirus HKU21. This discovery occurred three years after the description of the following three novel avian CoVs, munia coronavirus HKU13, thrush coronavirus 

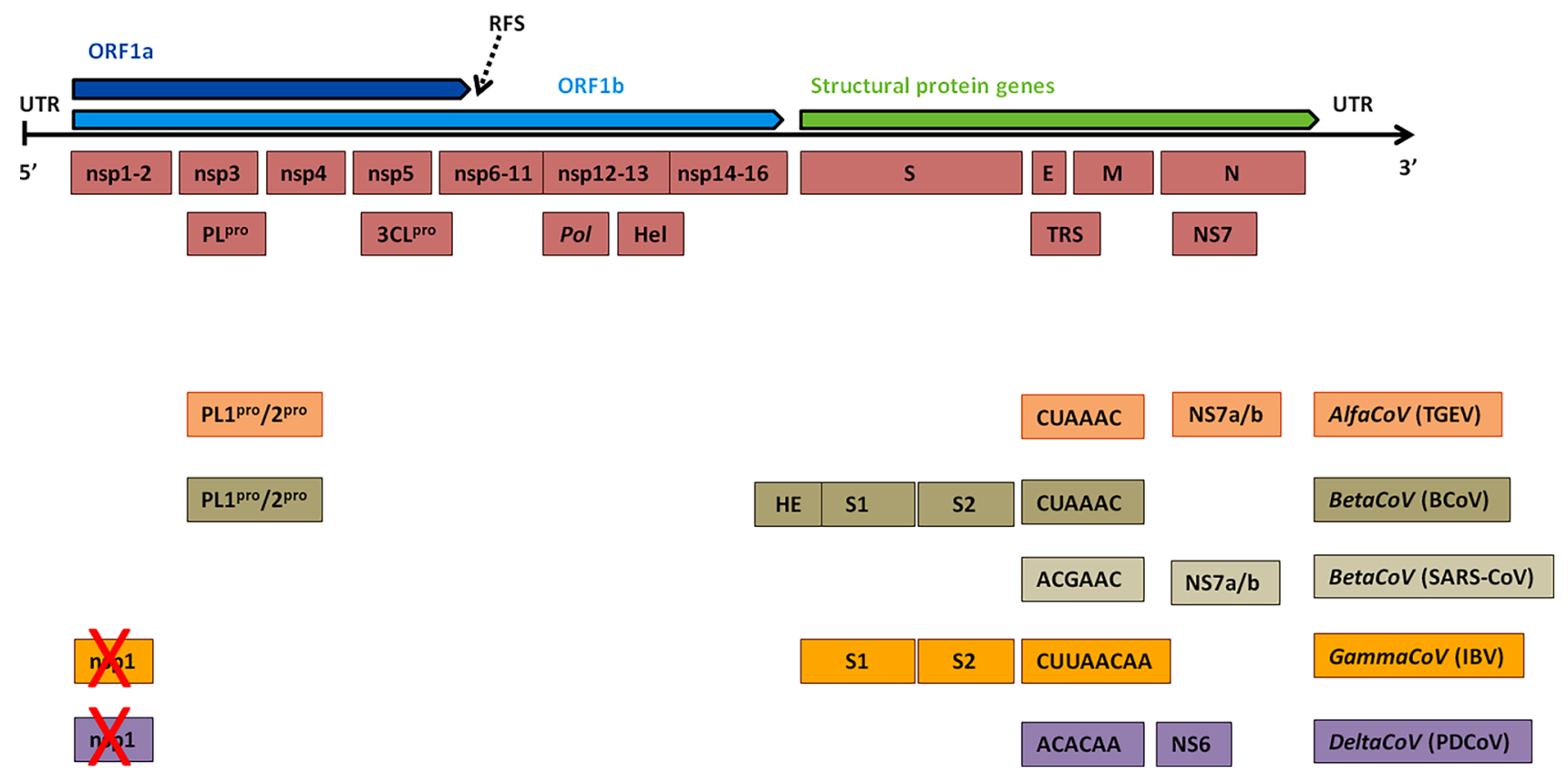

Fig. 2 Main genome regions of the four $\mathrm{CoV}$ genera belong to the subfamily Orthocoronavirinae in the family Coronaviridae. The 5' untranslated region (UTR), open reading frames (ORFs) $1 \mathrm{a} / \mathrm{b}$ encoding replicase polyproteins such as papain-like protease ( $\left.\mathrm{PL}^{\mathrm{pro}}\right)$, chymotrypsin-like protease (3CL), RNA-dependent RNA polymerase $(\mathrm{Pol})$, helicase (Hel), haemagglutinin esterase (HE) and functional

HKU12 and bulbul coronavirus HKU11 [44]. The genome sequencing showed that all these CoVs are very identity with the smallest genome compared with other $\mathrm{CoVs}$, and have an accessory gene like nonstructural protein 6 (NS6), as well as other accessory genes between the $\mathrm{M}$ and $\mathrm{N}$ genes; a stem-loop II motif (s2m) and the same putative TRS with the ACACCA sequence $[6,44,46]$. In addition, the genetic analyses of the whole genome based on PDCoV (Deltacoronaviruses genus) ORF1ab, S, M and N genes demonstrated high nucleotide identity, however, the comparison between these genes, $\mathrm{S}$ gene suggested more genetic diversity. In addition, all Chinese strains showed the same 3-nt (AAT, 19,473-19,477 nucleotide) deletion in the $\mathrm{S}$ gene confirming an evolutionary rate of $\mathrm{S}$ gene higher than PDCoV whole genome [6].

With the increasing of the CoV novel numbers that have recently been reported and the number of sequenced $\mathrm{CoV}$ genomes of each genus member after the emerged SARS, indicating a high diversity of $\mathrm{CoVs}$ based on phylogenetic and evolutionary rate, and their capacity of interspecies jumping [46]. Therefore, new outbreaks are expected and more often of zoonotic diseases such as the COVID-19 pandemic. This understanding of the structural and functional characteristics of the CoVs genome is essential to combat novel CoV infections in animals and humans [45]. nonstructural protein (nsp); and spike (S), envelope (E), membrane (M), nonstructural protein 6/7 (NS6/7), nucleocapsid (N), and 3' UTR are shown. Transcription-regulatory sequence (TRS). The ribosomal frameshift (RFS) site was indicated. One member of each genus is shown as a representative example. The figure is not drawn to scale

\section{Conclusions and future perspectives}

Nowadays, more than fifteen coronavirus-related diseases have been described in animals. The animal coronavirus infections have emerged in various species of mammals and birds and are generally associated with a wide spectrum of respiratory, intestinal and systemic infections. Furthermore, seven human coronaviruses have been described, of which four circulate seasonally and cause common colds, two other coronaviruses, SARS and MERS, have crossed from animal sources into humans but have not become endemic and recently SARS-CoV-2 is the causal agent of COVID-19 pandemic. The high mutation rate and recombination probability of CoVs allow the adaptation of them a wide variety of animals taking to new RNA viruses emerging in a short period time.

The coronavirus infections have highlighted the political concern, due to the serious social, economic, human health and animal health repercussions. Moreover, there are no measures to prevent and control a majority of these coronavirus infections. More economic investments by organizations and governments can also accelerate the implementation of measures like programs, policies, legislation and research in One Health perspective and the 


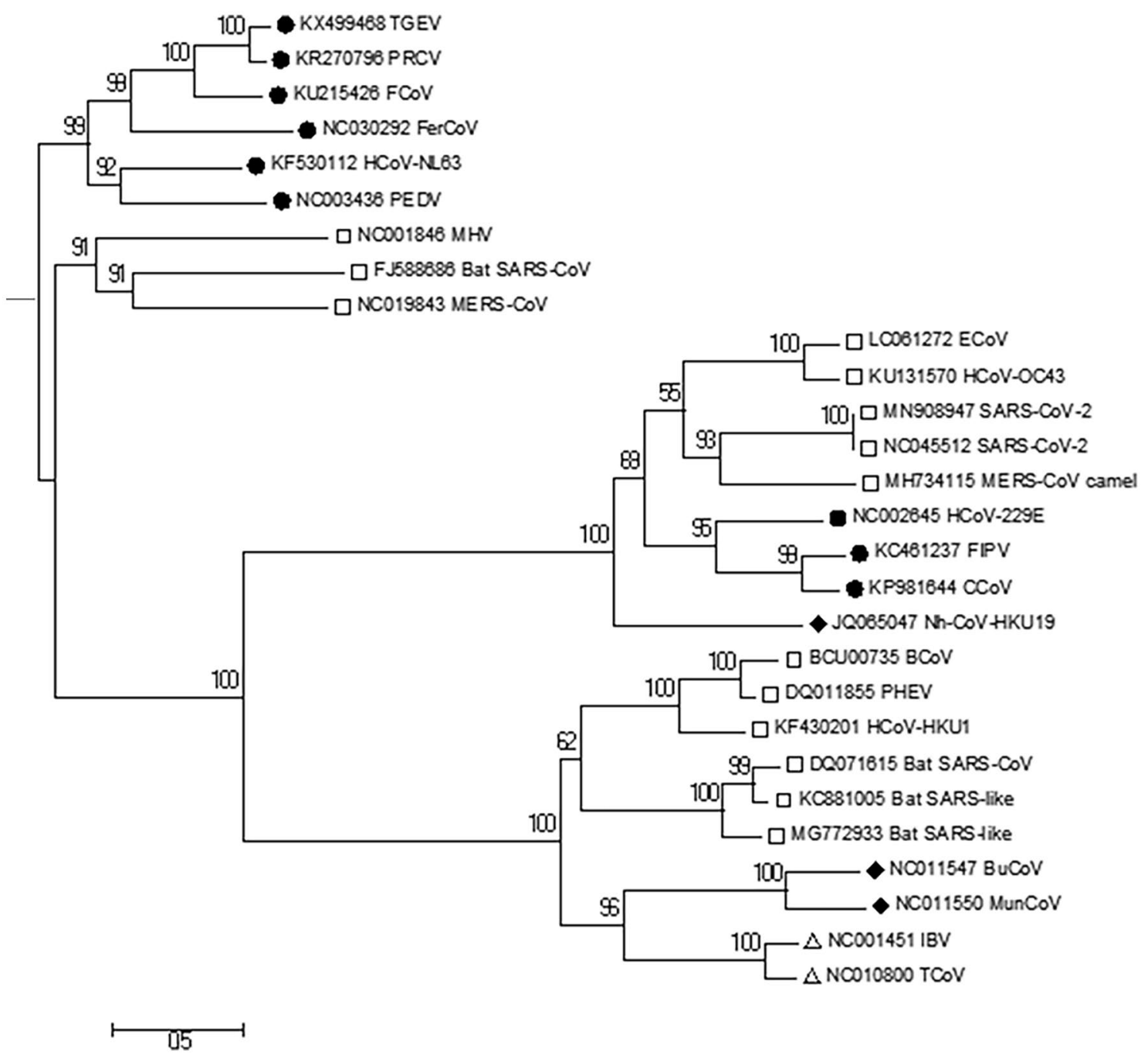

Fig. 3 Phylogenetic tree based on nucleotide sequences of complete genomes of $\mathrm{CoV}$ genera. This was constructed by the maximum likelihood method using the MEGA10 software package [49]. Bootstrap values were calculated based on 1000 replicates. The CoVs sequences

development of new strategies to control of diversity of the coronavirus diseases, such as vaccines and new drugs to their treatment.

Author contributions Methodology, CM and VS; validation, GI and PP; investigation, CM; resources, CM, VS, GI, PP; writing-original draft preparation, CM; supervision, GI and PP. All authors read and approved the final manuscript.

Funding This work was funded by the R\&D Project CAREBIO2comparative assessment of antimicrobial resistance in environmental biofilms through proteomics-toward innovative theranostic biomarkers, reference NORTE-01-0145FEDER-030101 and PTDC/ SAU-INF/30101/2017, financed by the European Regional Development Fund (ERDF) through the Northern Regional Operational Program (NORTE 2020) and the Foundation for Science and Technology (FCT). This work was supported by the Associate Laboratory for Green are deposited on GenBank database. The branch lengths are drawn to scale (number of substitutions per site). The coronavirus of the genus AlphaCoVs, BetaCoVs, DeltaCoVs and GammaCoVs are indicated filled circle, open square, filled square and open triangle, respectively

Chemistry-LAQV which is financed by national funds from FCT/ MCTES (UIDB/50006/2020 and UIDP/50006/2020). Vanessa Silva is grateful to FCT for her PhD Grant (SFRH/BD/137947/2018).

Data availability All data are included in the manuscript.

\section{Declarations}

Conflict of interest The authors declare that they have no conflict of interest.

Ethical approval This article does not contain any studies with human participants or animals performed by any of the authors.

Consent to participate Not applicable.

Consent to publish Not applicable. 


\section{References}

1. ICTV (2011) 9th Report. Coronaviridae. https://talk.ictvonline. org/ictv-reports/ictv_9th_report/positive-sense-rna-viruses2011/ w/posrna_viruses/222/coronaviridae. Accessed 13 Apr 2020

2. Weiss SR, Leibowitz JL (2011) Coronavirus pathogenesis. Adv Virus Res 81:85-164

3. Denison MR, Graham RL, Donaldson EF et al (2011) Coronaviruses: an RNA proofreading machine regulates replication fidelity and diversity. RNA Biol 8(2):270-279

4. Fehr AR, Perlman S (2015) Coronaviruses: an overview of their replication and pathogenesis. Methods Mol Biol 1282:1-23

5. MacLachlan N, Dubovi EJ (eds) (2016) Fenner's veterinary virology, 5th edn. Elsevier, New York

6. Zhang J (2016) Porcine deltacoronavirus: overview of infection dynamics, diagnostic methods, prevalence and genetic evolution. Virus Res 226:71-84

7. Tekes G, Thiel HJ (2016) Feline coronaviruses: pathogenesis of feline infectious peritonitis. Adv Virus Res 96:193-218

8. Acar DD, Stroobants VJE, Favoreel H, Saelens X, Nauwynck HJ (2019) Identification of peptide domains involved in the subcellular localization of the feline coronavirus $3 \mathrm{~b}$ protein. J Gen Virol 100(10): 1417-1430

9. Vijgen L, Keyaerts E, Lemey P et al (2005) Circulation of genetically distinct contemporary human coronavirus OC43 strains. Virology 337(1):85-92

10. Singhal T (2020) A review of coronavirus disease-2019 (COVID19). Indian J Pediatr 87(4):281-286

11. Larson HE, Reed SE, Tyrrell DA (1980) Isolation of rhinoviruses and coronaviruses from 38 colds in adults. J Med Virol 5(3):221-229

12. van der Hoek L, Pyrc K, Jebbink MF et al (2004) Identification of a new human coronavirus. Nat Med 10(4):368-373

13. Salata C, Calistri A, Parolin C, Palù G (2019) Coronaviruses: a paradigm of new emerging zoonotic diseases. Pathog Dis. https:// doi.org/10.1093/femspd/ftaa006

14. Guan Y, Zheng BJ, He YQ et al (2003) Isolation and characterization of viruses related to the SARS coronavirus from animals in southern China. Science 302(5643):276-278

15. Kellam P, Barclay W (2020) The dynamics of humoral immune responses following SARS-CoV-2 infection and the potential for reinfection. J Gen Virol 101(8):791-797

16. Martina BE, Haagmans BL, Kuiken T et al (2003) Virology: SARS virus infection of cats and ferrets. Nature 425:915

17. Chan-Yeung M, Xu RH (2003) SARS: epidemiology. Respirology. https://doi.org/10.1046/j.1440-1843.2003.00518.x

18. Chan JF, Lau SK, To KK et al (2015) Middle East respiratory syndrome coronavirus: another zoonotic betacoronavirus causing SARS-like disease. Clin Microbiol Rev 28(2):465-522

19. de Wit E, van Doremalen N, Falzarano D et al (2016) SARS and MERS: recent insights into emerging coronaviruses. Nat Rev Microbiol 14(8):523-534

20. Zhu N, Zhang D, Wang W et al (2019) (2020) A novel coronavirus from patients with pneumonia in China. N Engl J Med 382(8):727-733

21. Zhou P, Yang XL, Wang XG et al (2020) A pneumonia outbreak associated with a new coronavirus of probable bat origin. Nature 579(7798):270-273

22. CSG-ICTV (Coronaviridae Study Group of the International Committee on Taxonomy of Viruses) (2020) The species severe acute respiratory syndrome-related coronavirus: classifying 2019$\mathrm{nCoV}$ and naming it SARS-CoV-2. Nat Microbiol 5(4):536-44

23. Shi J, Wen Z, Zhong G et al (2020) Susceptibility of ferrets, cats, dogs, and other domesticated animals to SARS-coronavirus 2. Science 368(6494):1016-1020
24. Zhang T, Wu Q, Zhang Z (2020) Probable pangolin origin of SARS-CoV-2 associated with the COVID-19 outbreak. Curr Biol 30(7):1346-1351.e2

25. Almendros A, Gascoigne E (2020) Can companion animals become infected with Covid-19? Vet Rec 186(13):419-420

26. OIE (2020) World Organization for Animal Health. Questions and Answers on the 2019 Coronavirus Disease (COVID-19). https:// www.oie.int/scientific-expertise/specific-information-and-recom mendations/questions-and-answers-on-2019novel-coronavirus/. Accessed 18 Apr 2020

27. Deng J, Jin Y, Liu Y et al (2020) Serological survey of SARS$\mathrm{CoV}-2$ for experimental, domestic, companion and wild animals excludes intermediate hosts of 35 different species of animals. Transbound Emerg Dis 67(4):1745-1749

28. WHO (2020a) World Health Organization. Coronavirus disease (COVID-19) Pandemic. https://www.who.int/emergencies/disea ses/novel-coronavirus-2019. Accessed 02 Aug 2021

29. WHO (2020b) World Health Organization. COVID-19 diagnostic testing in the context of international travel. https://www.who. int/publications/i/item/WHO-2019-nCoV-Sci_Brief-internatio nal_travel_testing-2020.1. Accessed $20 \mathrm{Jul} 2021$

30. Xu C, Luo X, Yu C et al (2020) The 2019-nCoV epidemic control strategies and future challenges of building healthy smart cities. Indoor and Built Environ 29(5):639-644

31. WHO (2020c) World Health Organization. Clinical management of severe acute respiratory infection when COVID-19 is suspected. WHO/2019-nCoV/clinical/2020.4 https://www.who. int/publications-detail/clinical-management-of-severe-acute-respi ratory-infection-when-novel-coronavirus-(ncov)-infection-issuspected. Accessed 20 Apr 2020

32. The Novel Coronavirus Pneumonia Emergency Response Epidemiology (NCPERE) Team (2020) Vital surveillances: the epidemiological characteristics of an outbreak of 2019 novel coronavirus diseases (COVID-19)—China, 2020. China CDC Wkly 2(8):113-122

33. Chen N, Zhou M, Dong X et al (2020) Epidemiological and clinical characteristics of 99 cases of 2019 novel coronavirus pneumonia in Wuhan, China: a descriptive study. Lancet 395(10223):507-513

34. Yang X, Yu Y, Xu J et al (2020) Clinical course and outcomes of critically ill patients with SARS-CoV-2 pneumonia in Wuhan, China: a single-centered, retrospective, observational study. Lancet Respir Med 8(5):475-481

35. Huang C, Wang Y, Li X et al (2020) Clinical features of patients infected with 2019 novel coronavirus in Wuhan. China Lancet 395(10223):497-506

36. Zhou F, Yu T, Du R et al (2020) Clinical course and risk factors for mortality of adult in patients with COVID-19 in Wuhan, China: a retrospective cohort study. Lancet 395(10229):1054-1062

37. Woo PC, Lau SK, Chu CM et al (2005) Characterization and complete genome sequence of a novel coronavirus, coronavirus HKU1, from patients with pneumonia. J Virol 79(2):884-895

38. Sargiacomo C, Sotgia F, Lisanti MP (2020) COVID-19 and chronological aging: senolytics and other anti-aging drugs for the treatment or prevention of corona virus infection? Aging (Albany, NY) 12(8):6511-6517

39. Du B, Qiu HB, Zhan X et al (2020) Pharmacotherapeutics for the new coronavirus pneumonia. Zhonghua Jie He He Hu Xi Za Zhi 43(3):173-176

40. Saksena N, Bonam SR, Miranda-Saksena M (2021) Epigenetic lens to visualize the severe acute respiratory syndrome coronavirus-2 (SARS-CoV-2) infection in COVID-19 pandemic. Front Genet. https://doi.org/10.3389/fgene.2021.581726

41. Decaro N, Lorusso A (2020) Novel human coronavirus (SARSCoV-2): A lesson from animal coronaviruses. Vet Microbiol. https://doi.org/10.1016/j.vetmic.2020.108693 
42. Woo PC, Huang Y, Lau SK, Yuen KY (2010) Coronavirus genomics and bioinformatics analysis Viruses 2(8):1804-1820

43. Marra MA, Jones SJ, Astell CR et al (2003) The Genome sequence of the SARS-associated coronavirus. Science 300(5624):1399-1404

44. Woo PC, Lau SK, Lam CS et al (2012) Discovery of seven novel Mammalian and avian coronaviruses in the genus deltacoronavirus supports bat coronaviruses as the gene source of alphacoronavirus and betacoronavirus and avian coronaviruses as the gene source of gammacoronavirus and deltacoronavirus. J Virol 86(7):3995-4008

45. Snijder EJ, Decroly E, Ziebuhr J (2016) The nonstructural proteins directing coronavirus RNA synthesis and processing. Adv Virus Res 96:59-126

46. Woo PC, Lau SK, Huang Y, Yuen KY (2009) Coronavirus diversity, phylogeny and interspecies jumping. Exp Biol Med (Maywood) 234(10):1117-1127
47. Wu A, Peng Y, Huang B et al (2020) Genome composition and divergence of the novel coronavirus (2019-nCoV) originating in China. Cell Host Microbe 27(3):325-328

48. Narayanan K, Ramirez SI, Lokugamage KG, Makino S (2015) Coronavirus nonstructural protein 1: Common and distinct functions in the regulation of host and viral gene expression. Virus Res 202:89-100

49. Tamura K, Stecher G, Peterson D, Filipski A, Kumar S (2013) MEGA6: molecular evolutionary genetics analysis version 6.0. Mol Biol Evol 30:2725-2729

Publisher's Note Springer Nature remains neutral with regard to jurisdictional claims in published maps and institutional affiliations. 